\title{
Tacit Coordination and Equilibrium Selection in a Merit-based Grouping Mechanism: A Cross-cultural Validation Study
}

\author{
$\underline{\text { A. Gunnthorsdottir }}^{\mathrm{a}}$ and P. Thorsteinsson ${ }^{\mathrm{b}}$ \\ ${ }^{a}$ Vienna University of Economics and Business, and Australian School of Business \\ ${ }^{b}$ Department of Economics, University of Iceland, Reykjavik, Iceland \\ Email: A.Gunnnthorsdottir@gmail.com
}

\begin{abstract}
We test, via cross-cultural comparison, the robustness of complex tacit coordination in an endogenous-grouping mechanism. The mechanism requires coordination along two dimensions: 1) equilibrium selection and 2) the tacit coordination of an asymmetric equilibrium - a somewhat puzzling phenomenon commonly referred to as "Magic" (Kahneman, 1988, p. 12).
\end{abstract}

In 2010, Gunnthorsdottir et al. introduced and experimentally tested a dual-level extension of a standard Social Dilemma game, which they call "Group-based Meritocracy Mechanism" (GBM). The GBM incorporates group formation, which a standard Social Dilemma Game does not address. The GBM is, to our knowledge, the first formal model of contribution-based team formation. Formally incorporating group formation into a team game increases a model's external validity since under naturally occurring circumstances, most groups are consistently on the lookout for high-contributing members. Incorporating this feature into a team game however, complicates its equilibrium structure: A standard Social Dilemma's sole equilibrium of non-contribution by all still persists in the weaker form of a best-response equilibrium. There is now however also a second, very efficient equilibrium, which is payoff dominant, asymmetric, and not particularly obvious without a formal analysis. For this equilibrium to occur, experimental subjects must first select it over its alternative. If indeed selected, subjects must coordinate its asymmetric profile, via simultaneous decisions and without communication.

Kahneman (1988, p. 12) coined the term "Magic" for when experimental subjects coordinate an asymmetric equilibrium via simultaneous decisions, without communication. While Kahneman observed that the Nash Equilibrium is an excellent, albeit surprising, predictor of aggregate behavior in such a setting, he also noted that individual choices over trials were unpredictable. This fact adds to the puzzle of how such a high degree of aggregate coordination can be achieved at all. Kahneman initially observed the phenomenon in a Market Entry Game (MEG; Cary-Bobo, 1990; Selten and Güth, 1982), a simple game with a binary strategy space; its asymmetric equilibrium is both obvious and unique. Extensive experimental tests of "Magic" in MEGs show that it is a very robust phenomenon (for overviews, see, e.g., Camerer, 2003, Ch. 7.3; Ochs, 1999).

In experimental tests of the GBM, Gunnthorsdottir et al. (2010a; 2010b) report more striking "Magic" than previously reported: 1) since the GBM has two pure strategy equilibria, subjects first of all need to tacitly coordinate which equilibrium to select - the payoff dominant asymmetric one, or the payoff-inferior one of non-contribution by all. 2) The GBM's asymmetric equilibrium is not easy to detect. 3) While the MEGs strategy space is binary, in Gunnthorsdottir et al.'s version of the GBM and without loss of generality, the strategy space contains 101 choices, of which only two are part of the asymmetric payoff-dominant equilibrium.

Gunnthorsdottir et al.'s (2010a; 2010b) subjects, US university students, reliably selected the payoff dominant equilibrium and tacitly coordinated its asymmetric strategy profile. The current series of experiments test the robustness of these findings with additional tests in Iceland, a society with a distinctly different culture. Our results show that both the aggregate and individual behavioral patterns in both locations are very similar. While it is not yet clear how this phenomenon occurs, the findings here indicate that very efficient tacit coordination in contribution-based group formation is a robust occurrence.

Keywords: Endogenous grouping, public goods, mechanism design, experiment, tacit coordination 


\section{INTRODUCTION}

\subsection{Purpose}

The purpose of this paper is to establish, via a cross-cultural comparison, the robustness of the puzzling degree of coordinated decision-making in an endogenous grouping mechanism introduced by Gunnthorsdottir et al. (2010a; 2010b). In experimental tests of their "Group-based Meritocracy Mechanism" (GBM), Gunnthorsdottir et al. found that US students reliably selected, and accurately coordinated via simultaneous decisions and without communication, a payoff-dominant (Harsanyi and Selten, 1988) asymmetric equilibrium that is not easy to detect. While the payoff-dominant Nash equilibrium was a reliable organizing principle of aggregate behavior, individual decision-paths over rounds were unpredictable, so that the coordination task essentially presented itself anew in each round of the experiment.

\subsection{The Group-based Meritocracy Mechanism (GBM)}

The GBM is a multi-level extension of a standard linear Social Dilemma. It formally incorporates the important question of group formation, which regular Social Dilemma games bypass. A Social Dilemma's dominant strategy equilibrium is non-contribution by all. In contrast the GBM, where group membership is competitively based on contributions, has two equilibria in pure strategies. Its highly efficient payoffdominant equilibrium is asymmetric and rather non-obvious, yet US subjects coordinated it precisely and reliably, suggesting a natural and efficient response to merit-based social organization. The GBM involves simultaneous bi-level interaction, between all participants and within groups. We now describe each in turn:

\section{Level 1 Interaction: Between All Players, Competition for Team Membership}

All $\boldsymbol{N}$ society members decide simultaneously how much of their individual endowment $\boldsymbol{w}$ to keep for themselves, and how much to contribute to a group account. After having decided their group contribution, all $N$ players get ranked accordingly, with ties broken at random. Based on this ranking, participants are partitioned into $\boldsymbol{G}$ teams of size $\boldsymbol{n}$, so that the highest-ranking $n=N / G$ players are grouped together, then the next $n$ players, and so on. Based on this partitioning, players' earnings are computed as follows:

\section{Level 2 Interaction: Within Each Group, a Standard Social Dilemma Game}

Funds that participants keep for themselves get multiplied by $p$. Group account contributions are summed up over all $n$ group members, multiplied by $g$ and then disbursed equally to all members of the group, independent of their individual contribution. The ratio $g / n$ is the Marginal Per Capita Return (MPCR) from an individual contribution to the group account, henceforth denoted by $\boldsymbol{m}$. As long as $1 / n<m<p$, the game, were it not for the competitive Level 1 interaction, would be a standard linear Social Dilemma, where maximum efficiency is achieved if everybody contributes $w$ to the group account, while it is individually rational to contribute nothing. However, adding the Level 1 interaction changes the game's equilibrium structure:

\section{The GBM's Two Pure-Strategy Equilibria}

The GBM's two pure-strategy' equilibria differ greatly in efficiency. It is easily verified that noncontribution by all is a most inefficient best-response equilibrium. There is however, also a "Near-efficient Equilibrium" (NEE), which is payoff dominant, close to Pareto optimal, asymmetric, and not easy to detect. In a NEE, all society members contribute their entire endowment $w$ with the exception of $z<n$ players who contribute nothing. The exact value of $z$ depends on $g, n$ and $N$. The NEE asymptotically approaches full efficiency as the number of groups $G$ gets large. The NEE can be regarded as a loose model of successful meritocratic social organization, which is generally assumed to result in high levels of efficiency. Gunnthorsdottir et al. (2010a) show the NEE's existence and uniqueness, and provide the following Theorem that determines the value of $z$ for any set of parameters:

If $m \geq(N-n+1) /\left(N n-n^{2}+1\right)$, the GBM has a NEE in which $N-z$ players contribute $w$ and $z$ players contribute nothing.

$$
z \in[l, u] \text { is the integer where } l=(N-m N) /(m N-m n+1-m) \text { and } u=l+1
$$

As $G$ increases, the range of $m$ for which a NEE exists converges to the interval $(1 / n, 1)$. For a complete

\footnotetext{
${ }^{1}$ There also exist mixed-strategy equilibria. Gunnthorsdottir et al. (2010) document that subjects don't play these, providing empirical support for conjectures by theorists that mixing is counterintuitive if pure strategies are available (see, e.g., Kreps, 1990, pp.407-410; Aumann, 1985, p.19).
} 
proof, see Gunnthorsdottir, et al., 2010a; Gunnthorsdottir et al. 2010b provide a more general analysis encompassing two different levels of $w$.

\subsection{The Two Kinds of Coordination Required in the GBM}

The NEE puts high, and in fact dual, demands on subjects' coordinating abilities: 1) equilibrium selection and 2) the tacit coordination of an asymmetric equilibrium. We now briefly review experimental evidence from simpler experimental games, which require one or the other type of coordination. Our experimental results (Section 3) will reveal whether subjects, cross-culturally, are capable of dealing with the GBM's dual coordination requirements.

\section{Selecting from among Multiple Equilibria via Simultaneous Choice and without Communication}

If a game's Nash equilibrium is not unique, its predictive power is reduced. Additional criteria to identify the most desirable, likely or stable equilibrium among multiple equilibria have been proposed. Prominent among these refinements is Payoff Dominance, where a specific equilibrium should ensue since "commonly preferred" (Harsanyi and Selten, 1988, p. 81) so that each and every player is better off in the payoffdominant equilibrium than in any other.

Early results from order-statistic games with multiple symmetric Pareto rankable equilibria (see, e.g., Van Huyck et al., 1990, 1991; Cooper et al., 1990) indicated that subjects often fail to coordinate the efficient equilibrium and often minimize strategic risk instead. Later studies however indicated that certain additional features, lead to more efficient coordination. Some of these features also increase external validity. Some of these features happen to be built into the GBM, such as smaller group size, extensive feedback, shared experience (possibly including precedents established in other contexts), and a relatively unattractive secure option (e.g., Brandts and Cooper, 2006, 2007; see Devetag and Ortmann, 2007, for an overview of the literature)

- The GBM, while encompassing the "society" of all $N$ session participants, effectively partitions them into smaller teams where like-contributors are grouped together. This reduces contributors' strategic risk. Compare this to a standard coordination game where even a single slacker turns everybody else's superior effort into a loss.

- The feedback in GBM experiments is usually extensive (See Section 2.3; see Gunnthorsdottir, 2009, for feedback effects in the GBM).

- The GBM is a game of merit-based grouping, which is common in many naturally occurring circumstances; university entry rules or organizational hiring might serve as indirect precedents.

- The GBM's secure equilibrium of non-contribution by all is much less attractive than the NEE (See Section $3.1)$.

\section{Coordinating an Asymmetric Equilibrium via Simultaneous Choice and without Communication}

In 1988, Kahneman observed an experimental Market-entry Game (MEG, Cary-Bobo, 1990; Selten and Güth, 1982), a simple coordination game with a binary strategy space and a unique, intuitive asymmetric equilibrium in pure strategies. Puzzled by the apparent ease with this participants coordinated the equilibrium seemingly without learning or communication, and even though individual strategies over repeated trials seemed unsystematic, Kahneman $(1988$, p. 12) wrote, "[...] to a psychologist, it looks like magic". "Magic" in the MEG has shown itself to be a robust phenomenon in repeated experimental tests (see, e.g., Camerer, 2003, Ch. 7.3; Ochs, 1999). The question here is whether "Magic" can also be reliably observed under the increased demands for coordination posed by the GBM with a much larger strategy space, and an asymmetric equilibrium that is less obvious than the MEG's equilibrium, which furthermore is not unique.

\subsection{A Rationale for Cross-cultural Validation}

The experimental subjects of choice - or, rather convenience, have often been US university students. In order to increase the external validity of conclusions drawn from experiments, it is highly desirable to expand the subject pool. A small but growing literature addresses how culture or demographics impact interactive decision-making. The evidence is somewhat mixed: Experimental markets often lead to similar results internationally (See, e.g., Beaulier et al., 2004), but other games show noticeable variation; see, e.g., the classical early study by Roth et al. (1991) with Ultimatum Games (UGs). Differences in UG behavior become more pronounced the more cultures differ; see for example Henrich et al. (2002). Henrich et al. also cross-culturally test standard Social Dilemma games (of which the GBM is an extension) and find significant behavioral variation across distinct and diverse cultures. With regard to cross-cultural differences in the 
GBM, we have no a-priori hypothesis to go by. We are not aware of any work testing "Magic" crossculturally. On the other hand, culture might impact which of the two GBM equilibria subjects select.

\subsection{The US versus Iceland: Economy and Culture}

The two countries where our data sets are drawn from, the US and Iceland, are both developed economies and established democracies. They differ however along important dimensions including geography, demographics, economic structure, and hence, culture. In Iceland, a geographically isolated island nation, production is concentrated on a few sectors; its population of about 300,000 is highly homogeneous. The US has a population about 1000 times larger, and is ethnically, culturally and economically diverse.

\section{A Highly Individualistic, Masculine Society versus a Moderately Individualistic Feminine Society}

Figure 1 compares the two countries on Hofstede's (1980/2001) dimensions of culture. ${ }^{2}$ It can be seen that the US are a highly "Masculine" culture (MAS), while Iceland is the opposite, "Feminine". Masculine culture is among other things ego- and money-oriented, assertive and competitive (p. 297). High MAS might lead US subjects to vigorously compete for membership in high-contributing groups. The two countries also differ markedly on Individualism/Collectivism (IDV), that is, the degree to which citizens are integrated in groups, have close bonds with each other, and rely on each other. The US are the most individualistic nation world-wide with a score of 91 , while Iceland is estimated much lower, at 60 , even though still above the world mean of 43. (http://www.geerthofstede.com/hofstede united_states.shtml, Accessed 7 July 2011).

\section{A Meritocratic versus a Kinship-oriented Culture}

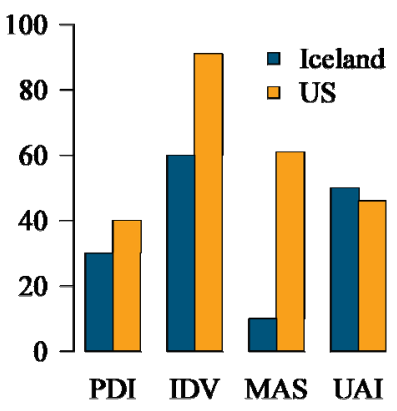

Figure 1.A comparison between Iceland and the US on Hofstede's dimensions of culture. PDI $=$ Power Distance, IDV =

Individualism, MAS = Masculinity, UAI = Uncertainty Avoidance.

Kinship clusters and other close personal or political ties strongly impact economic decisions in Iceland, including hiring or inter-firm interaction (Kristjansson, 2009; Baldvinsdottir, 1998). Compare this to the US with its nationally mobile work force and distinctly market-based exchange and hiring. ${ }^{3}$ Not surprisingly then, while both countries are meritocratic on a world-wide scale, Iceland ranks behind the US (Marks, 2010). Since the GBM is a simple model of meritocracy and since precedents impact equilibrium selection (Section 1.3), again, one might expect US subjects to respond more readily to the NEE's meritocratic incentives.

\section{EXPERIMENTAL METHOD}

\subsection{Subjects, Design and Parameters}

Participants were 48 George Mason University students, and 48 students from the University of Iceland. All were recruited from the general student population for a two-hour experiment, with a show-up fee of US\$ 7 or ISK 700 (116 ISK = 1 US\$), and further earnings contingent on decisions made by all session participants. ${ }^{4}$ Four sessions of 80 rounds each were conducted in each country. In each session there were $N$ $=12$ participants, in three groups of $n=4$. Each subject was endowed with $w=100$ integer tokens per round; $m$ was $0.5, p$ was set to 1 . The experimental instructions were in English in both locations and essentially the same, but their wording differed slightly between locations, with the instructions for Iceland slightly more precise. The reader can verify this at http://anna.rvik.com/GT/inUS.pdf (for the US) and at http://anna.rvik.com/GT/inice.pdf (for Iceland). ${ }^{5}$ The parameters, protocol, and subject interface were identical in both places. For US subjects, the exchange rate was 1000 tokens to 1 US\$ in three of the sessions, and 880 tokens to 1 US\$ in one session. Data from the latter did not differ from the other sessions. In Iceland, the conversion rate was 5.9 tokens to 1 ISK.

\footnotetext{
${ }^{2}$ In absence of direct Icelandic data, we work with estimates used by the ITIM consultancy and provided by G.J. Hofstede (personal communication, 2 July 2011).

${ }^{3}$ Despite the importance of personal ties in its economy, Iceland ranks as the seventh least corrupt country in the world on the Corruption Perception Index, while the US ranks $18^{\text {th }}$ (Zinnbauer and Dobson, 2009).

${ }^{4}$ The US data were previously published by Gunnthorsdottir et al. (2010a).

${ }^{5}$ We recognize that this may lead to a confound, but we do not consider this a major issue in this specific context. See the Conclusion.
} 


\subsection{Group Assignment}

In each round, after subjects had made their investment decisions, they were ranked according to their group contribution with ties broken at random, and partitioned in three groups of four. Their earnings for this round were calculated based on the group to which they had been assigned. Subjects were regrouped according to this criterion in each decision round.

\subsection{End of Round Feedback}

At the end of each round, a subject's computer displayed her private and group investment in that round, the total investment made by the group she had been assigned to, and her total earnings. Each subject also saw an ordered series of the current round's group account contributions by all $N$ session participants, with a subject's own contribution highlighted. The ordered series was split into three groups of four so that subjects would see that they had been ranked and grouped according to their contributions with ties broken at random.

\section{RESULTS AND DISCUSSION}

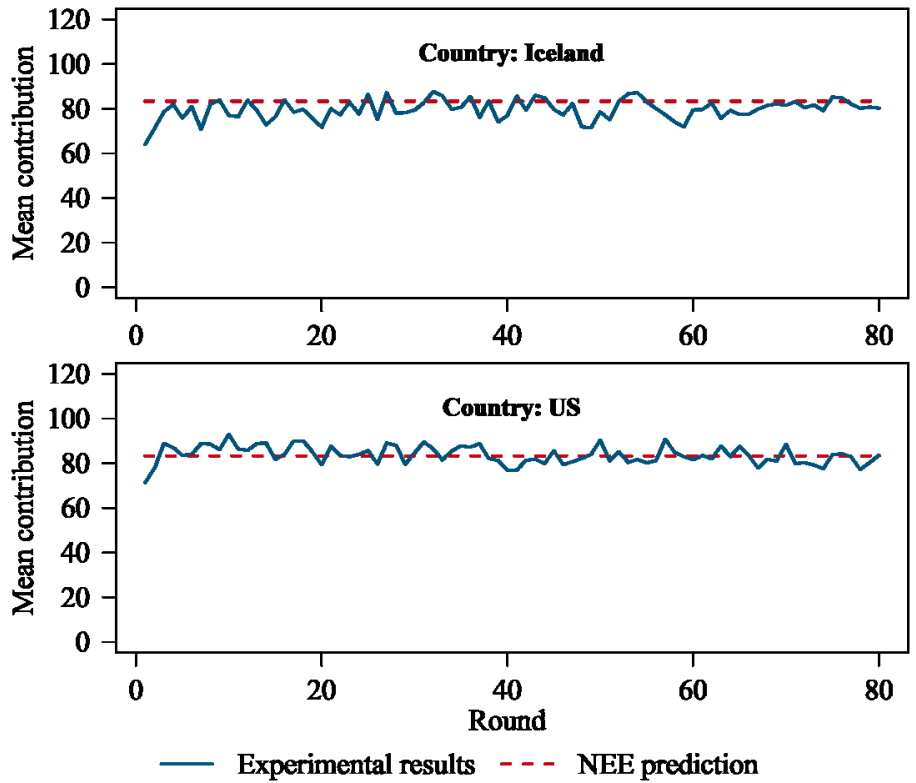

Figure 2. Mean contribution per round in each country.

\subsection{The NEE Predicts Aggregate Behavior}

Inserting the experimental parameters (Section 2.1) into the Theorem (Section 1.2), it can be verified that $z=2{ }^{6}$ so that ten out of twelve players contribute fully, while two contribute nothing. A non-contributor's NEE earnings are 200 tokens; a contributor's expected NEE earnings are 180 tokens. The NEE mean contribution per round is 83.3 tokens (the straight dashed line in Figure 2). The NEE is clearly "commonly preferred" over the other, inefficient equilibrium by about a factor of two, and is thus much more attractive than the secure alternative. ${ }^{7}$ This should facilitate NEE coordination in spite of the strategic risk involved.

The solid lines in Figure 2 show the mean contribution per round, separately for each country. Both Icelandic and US subjects closely follow the NEE mean. Even though the NEE is unlikely to be obvious to subjects, the vicinity of the NEE mean is reached very quickly in both countries. Icelandic subjects contributed on average 78.56 tokens out of 100 already in Round 3 and 81.94 tokens in Round 4; US subjects contributed 77.81 in Round 2 and 88.94 in Round 3.

\subsection{The Frequencies with which Strategies were Chosen Correspond to the NEE Frequencies}

Figure 3 shows the frequencies of observed strategies by country. Squares represent the NEE proportions. Frequencies are very close to NEE predictions in both countries; again, the US data are slightly more precise.

\subsection{A Barely Noticeable Learning Trajectory in Iceland and no Apparent Learning in the US}

Icelandic subjects exhibited a slight learning process over rounds as they increased their precision over subsequent rounds. Their mean contribution over 80 rounds and four sessions is 79.61 tokens; the corresponding US value is 83.74, essentially exactly the NEE mean. A Mann-Whitney-Wilcoxon test (see, e.g., Siegel and Castellan, 1988) with the session mean as the unit of analysis indicates that this slight difference is systematic (Mann-Whitney $\mathrm{U}=10, \mathrm{n}=\mathrm{m}=4, \mathrm{p}[2$-tailed] $<.03$ ). However, the difference gradually disappears over rounds: When session means are based on Rounds 21-80 only, one can no longer reject the null hypothesis that the session means are drawn form the same population (Mann-Whitney $U=13$, $p[2$ -

\footnotetext{
${ }^{6}$ Plugging the experimental parameters into the Theorem it can be seen that a NEE exists for the experiment's parameters. Further, it can be verified that for these parameters the upper and lower bounds of $z$ are 2.33 and 1.33 respectively, so that $z=2$.

${ }^{7}$ Recall that an individual's secure equilibrium earnings are only $w=100$.
} 
tailed] <.2). In the last 20 rounds the Icelandic overall mean is 81 and the US overall mean is 82 . Both countries, in spite of their cultural differences, clearly coordinate the NEE rather than the inefficient equilibrium. However, while Icelandic students are used to reading English, it is not their native language. Gunnthorsdottir (2009) suggests that the precision with which the NEE is coordinated is, sensitive to subjects' knowledge of the rules of the game.

\subsection{Individual Decision Paths are Unsystematic}

While in the aggregate subjects follow the NEE closely the same cannot be said for individual subjects. Graphs of all individual decision paths from both countries can be viewed http://anna.rvik.com/GT/ind.pdf. In both countries, many players oscillate unpredictably between the two NEE strategies of contribution and noncontribution. While individual decision paths exhibit seemingly erratic oscillations, the aggregate paths (Figure 2) remain quite smooth. This can only occur if individual oscillations offset each other. A similar pattern has also been observed in Market Entry Games (See Section 1.3).

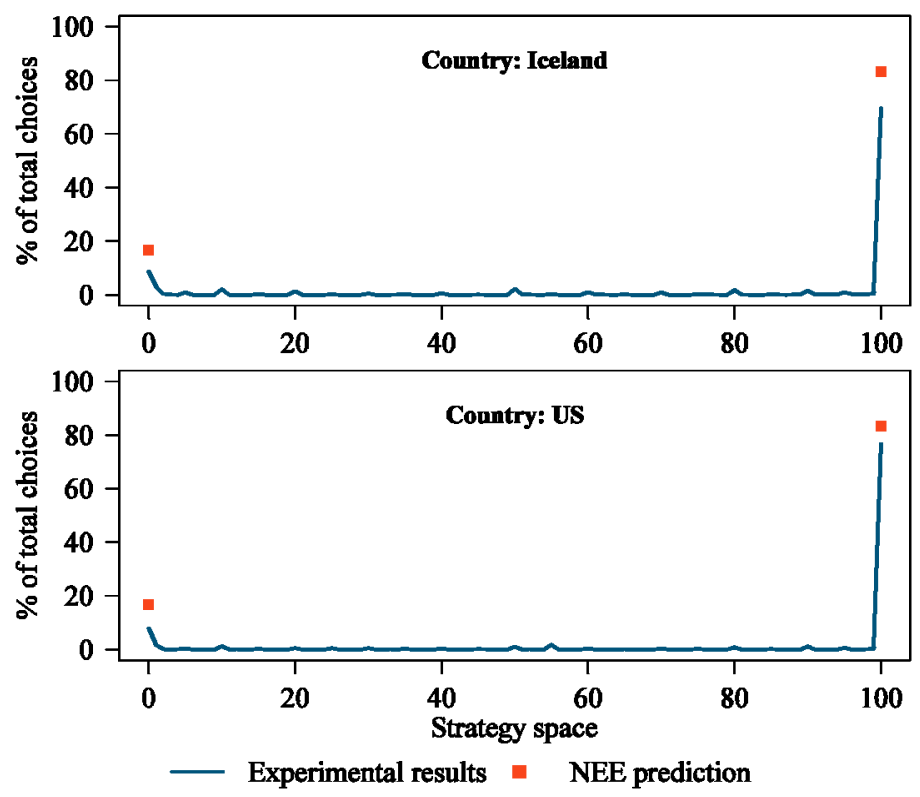

Figure 3. Strategy choices over four sessions and 80 rounds.

\section{CONCLUSION}

We have examined the robustness of Payoff Dominance and tacit asymmetric equilibrium coordination in a GBM mechanism with data from two different societies that differ in their culture. The GBM presents a stringent test of human abilities at tacit coordination, since it requires coordination along two dimensions,

- Equilibrium selection

- If Payoff Dominance holds, the coordination of an asymmetric strategy profile.

The following aspects of our findings underscore participants' striking and cross-cultural ability at tacit coordination provide strong empirical support for the Nash equilibrium as an organizing principle of behavior even if it is asymmetric and somewhat obscure:

1. GBM subjects coordinate, with great precision, an asymmetric payoff-dominant equilibrium that they are very unlikely to be able to consciously detect

2. While payoff-dominant, the Near-efficient Equilibrium (NEE) equilibrium is not fully efficient. In spite of the competitiveness of the situation, full efficiency does not ensue even in a highly competitive culture (the US); full contribution by all would simply not be in equilibrium.

3. The surprising ability to coordinate the GBM's payoff-dominant asymmetric equilibrium is a robust occurrence: subjects in different countries and even with slightly differently worded instructions, produce identical aggregate patterns of behavior via simultaneous decisions, without communication and without a significant learning trajectory.

\section{Future research}

As outlined in Section 1.5, Iceland and the US differ on important cultural and social dimensions. However, both are democratic and affluent Western societies. Gunnthorsdottir et al. (2010b) suggest that the GBM could serve as a simple formal model of meritocratic social grouping. While the US is more meritocratic than Iceland (Section 1.5), both countries are somewhat close on this dimension. Further, subjects in both locations were university students used to some merit-based selection. Real-life precedents may thus have helped these subjects coordinate the NEE rather than the alternative inefficient equilibrium. The next step is to test the robustness of our findings here with streamlined instructions and at the same time different demographic groups, in developing countries or native communities, and in communities where social organization is less meritocratic and more privilege-based. 


\section{REFERENCES}

Aumann, R. (1985). What is game theory trying to accomplish? In Arrow, K., Honkapohja, S. (Eds.), Frontiers of Economics. Blackwell, Oxford, pp.909-924.

Baldvinsdottir, H. D. (1998). Networks of Financial Power in Iceland: The Labour Movement Paradox. Ph.D. thesis, Lancaster University. Retrieved from: http://www.networksofpower.net/

Beaulier, S., Boettke, P. and Rathbone, A. (2004). Efficiency in markets: evidence from classroom experiments with a cross-section of students from East and Central European countries, and the former Soviet Union. Mercatus Center Working Paper 27.

Brandts, J. and Cooper, D.J. (2006). A Change Would Do You Good...An Experimental Study on How to Overcome Coordination Failure in Organizations. American Economic Review, 96, 669-693.

Brandts, J. and Cooper, D.J. (2007). It's what you say, not what you pay: an experimental study of manageremployee relationships in overcoming coordination failure. Journal of the European Economic Association 5, 1223-1268.

Camerer, C. (2003). Behavioral Game Theory. Princeton, NJ: Princeton University Press.

Camerer, C. and Fehr, E. (2006). When does "economic man" dominate social behavior? Science 311, 47-52.

Cooper, R., DeJong, D., Forsythe, R. and Ross, T. (1990). Selection criteria in coordination games. Some experimental results. American Economic Review, 80, 218-233.

Gary-Bobo, R.J. (1990). On the existence of equilibrium points in a class of asymmetric market entry games. Games and Economic Behavior 2(2), 239-246.

Devetag, G. and Ortmann, A. (2007). When and why? A critical survey on coordination failure in the laboratory. Experimental Economics 10, 331 - 344.

Gunnthorsdottir, A. (2009). Equilibrium and type: The crucial role of information. In Anderssen, R.S., R.D. Braddock and L.T.H. Newham (Eds.) 18th World IMACS Congress and MODSIM09 International Congress on Modeling and Simulation. Modeling and Simulation Society of Australia and New Zealand and International Association for Mathematics and Computers in Simulation, July 2009, pp. 1450-1456. ISBN: 978-0-9758400-7-8. http://www.mssanz.org.au/modsim09/D9/gunnthorsdottir.pdf

Gunnthorsdottir, A., Vragov, R., Seifert, S. and McCabe, K. (2010a). Near-efficient equilibria in contribution-based competitive grouping. Journal of Public Economics 94, 987-994.

Gunnthorsdottir, A., Vragov, R., and Shen, J. (2010b). Tacit coordination in contribution-based grouping with two endowment levels. In R.M. Isaac \& D. A. Norton (Eds.), Research in Experimental Economics, $13,13-75$.

Harsanyi, J. and Selten, R. (1988). A General Theory of Equilibrium Selection In Games. MIT Press, Cambridge, MA.

Henrich, J. Boyd, R., Bowles, S., Camerer, C., Fehr, E., Gintis, H. and McElraeth, R. (2001). In search of homo economicus: Behavioral experiments in 15 small-scale societies. American Economic Review 91, 73-77.

Hofstede, G. (1980). Culture's Consequences, $2^{\text {nd }}$ ed. London: Sage.

Kahneman, D. (1988). Experimental economics: a psychological perspective. In Tietz, R., Albers, W., Selten, R. (Eds.), Bounded Rational Behavior in Experimental Games and Markets. Springer, Berlin, 11-18.

Kreps, D.M.,(1990). A Course in Microeconomic Theory. Princeton University Press, Princeton NJ.

Kristjansson, G. H. (2009). Pólitískar stöðuveitingar á Íslandi.Stjórnmal og Stjórnsýsla.

Marks, G. (2010). Meritocracy, modernization and students' occupational expectations: Cross-national evidence. Research in Social Stratification and Mobility 28, 275-289.

Ochs, J. (1999). Coordination in market entry games. In Budescu, D., Erev, I., Zwick, R. (Eds.), Games and Human Behavior: Essays in Honor of Amnon Rapoport. Erlbaum, Mahwah NJ, pp.143-172.

Roth, A.E., Prasnikar, V., Okuno-Fujiwara, M., and Zamir, S. (1991). Bargaining and market behavior in Jerusalem, Ljubiljana, Pittsburgh, and Tokyo: An experimental study. American Economic Review 81, 1068-1095.

Selten, R., and Güth, W. (1982). Equilibrium point selection in a class of Market Entry games. In Deistler, M., Fürst, E. and Schwadlauer, G. (Eds.), Games, Economic Dynamics, and Time Series Analysis: A Symposium in Memoriam Oskar Morgenstern. Physica, Vienna, pp.101-116.

Siegel, S. and Castellan, N.J. (1988). Nonparametric Statistics for the Behavioral Sciences (2 ${ }^{\text {nd }}$ ed.). NY: McGraw-Hill.

Van Huyck, J., Battalio, R. and Beil, R.O. (1990). Tacit coordination games, strategic uncertainty, and coordination failure. American Economic Review 80, 234-248.

Van Huyck, J., Battalio, R. and Beil, R.O. (1991). Strategic uncertainty, equilibrium selection principles, and coordination failure in average opinion games. Quarterly Journal of Economics 106, 885-911.

Zinnbauer, D., \& Dobson, R. (2009). Global Corruption Report 2009: Corruption and the Private Sector. Transparency International (p. 492). New York. 